\title{
An Introduction to Biophysics
}

\author{
Moganty R. Rajeshwari, 2013, Rastogi Publications, New Delhi. pp. 1-368 (Price 275.00) \\ ISBN978-93-5078-015-2
}

Biophysics is the science of the application of the laws of physics to biological phenomena. To be precise, it is an interdisciplinary science using methods of and theories from physics to study biological systems. It spans all scales of biological organization, from molecular to whole organisms and ecosystems. Biophysics has fascinated both basic and applied scientists alike. However, in spite of the fact that excellent research work was being done at some institutes in the early sixties, only a few universities in India, such as All India Institute of Medical Sciences, Delhi; Bombay University; Punjab University, Chandigarh and Saha Institute of Nuclear Physics, Calcutta had a Biophysics department, awarding undergraduate and post-graduate degrees in the discipline. Moreover, the curriculum at each place was different depending on the interests of the founders. There was a lack of a uniform text book in Biophysics although, a few excellent monographs and review articles on biophysical techniques, radiation and membrane biophysics were available. Also, many biochemistry books covered chemical and some structural aspects of bio-molecules. These books served as excellent help to researchers with postgraduate degrees in basic physics, chemistry or medical sciences. But a book, which a high school pass-out entering the discipline of biophysics needed, was not easily available till the end of the last century. Another problem was the high cost of the available books. During the last one decade, a couple of Biophysics books focussing on different areas of the field have become available.

The book "An Introduction to Biophysics" by Prof. Moganty R. Rajeshwari serves as an excellent introductory textbook for students e $\mathrm{n} \mathrm{t}$ e $\mathrm{r}$ i $\mathrm{n} \mathrm{g}$ biophysics. What I liked about the book is its simplicity and assumption of no prior knowledge of advanced topics such as $\mathrm{q} \mathrm{u}$ a $\mathrm{n} \mathrm{t} \mathrm{u} \mathrm{m}$

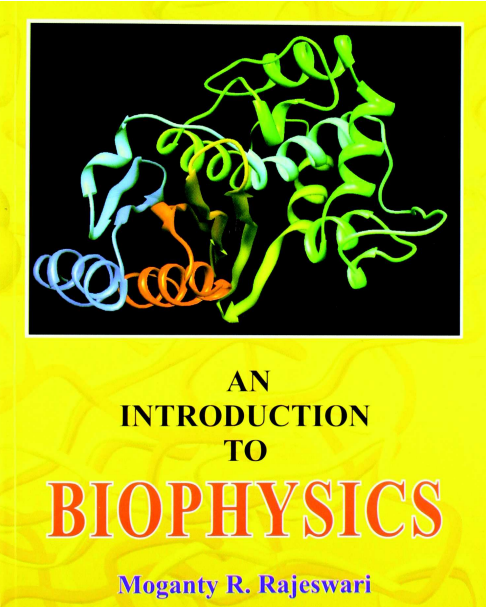
mechanics. The

book consists of 368 pages and is available for mere Rs 275. It has four units: I-Basic Biophysics, IIThermodynamics, Bioenergy and Membrane Biophysics, III-Biophysical Techniques and IVEnvironmental Biophysics. It covers important areas in basic biophysics, bioenergetics, radiation and membrane biophysics and gives the students adequate knowledge about the use of biophysical techniques. The book familiarises them with the most advanced concepts in structural and functional biophysics and prepares them for research in structure, function and interactions of biomolecules.

Unit I of the book starts with basic concepts in atomic structure, wave theory and uncertainty principle. These topics are essential for understanding different spectroscopy and diffraction techniques. The Unit also elaborates on 'water', the simplest and most important molecule for life. It discusses at length the laws of thermodynamics which are needed for understanding of bioenergetics, role of ATP, oxidation reduction principle and thermo regulation. 
Unit-II is dedicated to thermodynamics, bioenergy and biomembrane. I do not personally agree with the title of the unit. As per its contents, it could have been simply 'Biomolecules'. However, the title does not undermine the importance of this section. Dr. Rajeswari, herself being a structural biologist working in the area of DNA and Protein and their interactions with other molecules, has adequately explained macromolecular (carbohydrates, proteins, nucleic acids and lipids) structures and interactions. I read with great interest the portion dealing with structural polymorphism of DNA (A, B, C, D and $\mathrm{M})$, and unusual and Multi-Stranded DNA structures. The author has detailed on the nomenclature for atoms in nucleotide and definition of torsional angles $(\alpha, \beta$, $\gamma, \delta, \varepsilon, \zeta, \chi)$. Equally interesting is her chapter on protein structure. Starting from amino acids, their chemical structures, ionization, definition of torsional angles $\phi$ and $\psi$, peptide bond, Ramachandran Plot and its importance in structural organization she elaborates on different levels of protein structures, structural motives and protein folding. The chapter ends with DNA-Protein interaction, and structure and aggregation of lipids. There is a separate chapter on membrane biophysics.

Unit-III relating to biophysical techniques goes over to density and charge based separation techniques such as viscometry, centrifugation, chromatography, SDS-PAGE and mass spectroscopy. Her description of spectroscopic techniques (UV-VIS, flourescence, CD, ORD, vibrational and IR, and Raman) is most useful for the students, because, for each technique, she has given practical examples. How these methods can be applied for understanding biomolecular structure, conformation and interactions is explained meticulously. She has also introduced structure determination techniques as: X-ray diffraction crystallography, Nuclear Magnetic Resonance (NMR) and 2D NMR. Although quite basic, the portion on atomic absorption spectroscopy, flame photometry and light microscopy is needed for completion of the Unit.
Unit-IV is dedicated to environmental biophysics consisting of radiation biophysics and photosynthesis. It completes the linkage between atomic and molecular biophysics to the ecosystem and environment. One important thing about the book is a set of questions and hints when necessary, at the end of each section. Also, there is a section on reading material.

There are some shortcomings in the book. I found it difficult to follow topics from the "Contents" page because of the absence of page numbers and the numbering system for the chapters. Although different publishers use different numbering methods, I still think the decimal system is the best for such a complex book with four units, each of which is divided into several chapters which further consist of several headings and sub-headings.

Secondly, although the author has done a great job of starting from atomic structure and basic quantum mechanics and connecting them to molecular orbital (MO) theory, a modern biophysicist needs a little more. Different MO methods such as AM1, PCILO, Gaussian, MOPAC, EHMO etc used by popular modelling packages as MOE (Molecular Operating Environment) or HYPERCHEM could have been added briefly in a page or two. I also think that an introduction to force fields, definition of torsional angle and nonbonded potential used for computing Ramachandran Plot should be included in a biophysics book. Thirdly, the graphics and illustrations at many places could have been better, since this forms an important portion of any biophysics book. For example, one can have better illustrations for $\mathrm{L}$ and $\mathrm{D}$ amino acid concept in figure 4.11, backbone torsional angles in peptide, peptide bond, phospholipid nomenclature than figures 4.16 and 4.17. The book contains some colour plates but a few more colour pictures to illustrate lipid organization, structural domains in proteins, DNA polymorphism and tRNA structure could enhance the understanding of the structures. However, these points do not undermine the value of book as they can be addressed in the 
future edition of the textbook.

While teaching Biophysics for over forty years, I found lack of proper text book as a big obstacle in my efforts. I would have no hesitation in prescribing this book to my students. It is complete from all points of view and serves as an important link for a new entrant in Biophysics to connect to the world of structure, function and interaction of biomolecules to understand environment and our ecosystem.

Dr. Mrs. Vidya Kothekar, Ph.D., F.N.A.Sc Former Professor of Biophysics, All India Institute of Medical Sciences, New Delhi

Former Director, Dr. D. Y. Patil Biotechnology \& Bioinformatics Institute, Pune Former Professor of Bioinformatics \& Head, Biotechnology, Jaypee Institute of Information Technology, Noida, UP

Address: Flat No 19, CONIFER, Marutrao Gayakwad Nagar, D. P. Road, Aundh, Pune 411007 Ph: 02025887025, 7350909600 (M)

E-mail: vidyakothekar@gmail.com 